This item was submitted to Loughborough's Research Repository by the author.

Items in Figshare are protected by copyright, with all rights reserved, unless otherwise indicated.

\title{
Dynamic thermal simulation models of buildings: a new method for empirical
} validation

PLEASE CITE THE PUBLISHED VERSION

http://dx.doi.org/10.1177/014362449101200101

PUBLISHER

(c) SAGE Publications

VERSION

VoR (Version of Record)

\section{PUBLISHER STATEMENT}

This work is made available according to the conditions of the Creative Commons Attribution-NonCommercialNoDerivatives 4.0 International (CC BY-NC-ND 4.0) licence. Full details of this licence are available at: https://creativecommons.org/licenses/by-nc-nd/4.0/

\section{LICENCE}

CC BY-NC-ND 4.0

\section{REPOSITORY RECORD}

Lomas, Kevin J.. 2019. "Dynamic Thermal Simulation Models of Buildings: A New Method for Empirical Validation”. figshare. https://hdl.handle.net/2134/17455. 
Summary A new empirical validation methodology for dynamic thermal models has been devised and illustrated using the three models ESP, SERIRES and HTB2, and a simple data set collected from the Polytechnic of Central London test cells. The method hinges on making an initial base case prediction and then taking account of the errors and uncertainties in a systematic way. The method proved capable of revealing internal errors in thermal models. The relationship of this method to other validation techniques is discussed.

\title{
Dynamic thermal simulation models of buildings: New method for empirical validation
}

\author{
K J Lomas BSc PhD \\ School of the Built Environment, Leicester Polytechnic, PO Box 143, Leicester, LE1 9BH, UK
}

Received 12 February 1990, in final form 18 June 1990

\section{Introduction}

In the United Kingdom a joint Building Research Establishment (BRE) and Science and Engineering Research Council (SERC) programme to investigate analytical and empirical validation techniques for dynamic thermal models of buildings ${ }^{\text {(1) }}$ has recently been completed. The final report runs to over six volumes and covers techniques based on analytical verification, intermodel comparisons and empirical validation as well as the application of sensitivity analysis, parametric studies and other novel statistical techniques. The author (SERC funded) was principally concerned with empirical validation, that is, techniques based on comparing model predictions with measurements made in real buildings $s^{(2)}$.

To evaluate the accuracy of the underlying theoretical basis of the models, validation téchniques must be capable of revealing the presence of internal errors (in the algorithms and numerical processes used by the model). To do this it is essential that external errors (in the model input data, building response data and measured weather data) are minimised or controlled. The potential power of empirical validation means that it is widely used for validating dynamic thermal models; over 130 comparisons between actual building performance and predictions made by thermal models have been disclosed by a recent literature survey ${ }^{(3)}$. However, a review of previous validation work involving $\mathrm{BLAST}^{(4)}$, DEROB $^{(5)}, \operatorname{ESP}^{(6)}$ and SERIRES ${ }^{(7)}$ concluded that the effects of (external) errors have meant that none of the previous empirical studies would have produced conclusive evidence of internal errors in the models themselves ${ }^{2(8)}$. The following problems were identified:

(a) A validation methodology was not devised and clearly stated before performing the validation studies.

(b) The data sets used often contained large unquantifiable sources of external errors which were not accounted for in a rational way by the validation procedures adopted.

(c) The validation methods contained no mechanism for identifying the underlying reason for divergence between model predictions and measured data.

The intention of this paper is to propose and then test a new methodology which tackles problems $(b)$ and $(c)$ and which is sufficiently robust that it may be used with the widest possible range of models and data sets. The methodology is tested using three models ESP, HTB2 ${ }^{(9)}$ and SERIRES. Because these models operate at the detailed mechanistic level they are particularly demanding in their data input requirements. Therefore, validation techniques which are adequate for validating these models are also likely to be sufficient to test less sophisticated (lumped parameter) models.

The data set is from a simple direct-gain test cell, monitored by the Polytechnic of Central London. This was identified as one of only six sites throughout the world where structures have been built and monitored in such a way that the data may be valuable for validating a range of thermal models ${ }^{(3)}$. The cells were designed primarily to evaluate alternative passive solar features, for application in domestic buildings. Test cells represent a compromise between the conflicting demands for reality (i.e. monitoring actual domestic or commercial buildings) and the needs of experimental control. The PCL cells, in particular, have a tighter construction, a smaller volume, and a much higher proportion of the fabric heat loss is through the glazing. The data gathered stress the algorithms dealing with thermal storage, solar transmission and thermal conduction through windows. For model validation it is particularly useful to have data such as these which stress a few key algorithms, rather than data from situations in which the effects of many algorithms may have a similar weighting.

More recently the proposed validation methodology has been used with data relating to real, occupied, intermittently heated buildings. It is proving robust enough even under these demanding conditions. This work will be discussed elsewhere.

\section{Proposed validation methodology}

Most frequently, validation is approached by comparing predictions with measured data. If mismatches are found the input data are changed, within the bounds of plausibility, to improve the fit; this cycle may be repeated a number of times. This will be termed a Class B approach. Virtually all the validation exercises uncovered by the recent literature search $^{(3)}$ followed this approach, although it was not overtly declared as the modus operandi. The approach invariably led to close agreement between the predictions and measure- 
ments, so the model was claimed to be validated. In fact, all this method may prove is that with carefully chosen input data the model is capable of reproducing the observed phenomena (see section 7 below).

In the design situation the input data must be selected, predictions made, and decisions taken, without the benefit of knowing the actual performance of the building. It is much more relevant therefore to adopt a Class A approach to validation. This involves firstly modelling the structure as accurately as possible, taking care not to introduce any external errors by making approximations or blunders. Then, the predictions must be compared with the measurements without making refinements or repeating simulations. The difference between the measurements and predictions is then a true measure of the accuracy possible under conditions approaching those in which the model will be used in practice. Ideally, the predictions should be made in ignorance of the measure results and certainly no attempt should be made to manoeuvre a good fit between the measurements and the predictions (e.g. by manipulating the model input data). These are termed the 'base-case predictions', and they remain fixed throughout the remainder of the validation process.

The uncertainties in the base-case predictions are then accounted for, in a logical and systematic way, by quantifying the magnitude of all the errors in both the measurements and the predictions. The measurements and predictions are then compared statistically taking these errors into account.
This approach leads to a three-tier empirical validation methodology (Figure 1).

(a) Level 1: A base-case prediction is obtained without regard to the measured performance. These predictions, and the corresponding measurements, are then compared and if they differ by less than the errors in the measurements alone, the model is deemed to be satisfactory at Level 1 for the particular situation examined; if not, it is advisable to progress to Level 2.

(b) Level 2: The total uncertainty in the predictions due to external errors in the model input data, is quantified. If the base-case predictions for the parameters of interest differ from the measurements by less than the total uncertainty, the model is deemed to be satisfactory at Level 2 for the particular situations studied; if not, it is useful to progress to Level 3.

(c) Level 3: The internal errors which cause the divergent predictions are detected, either by comparing the predictions of individual algorithms with detailed mechanism level data, or by using some other validation techniques.

Having completed Level 3 , it will be possible to rectify the internal errors and repeat the validation process.

If a model is deemed to be satisfactory at either Level 1 or Level 2, this does not mean that there are no internal errors. Rather, any errors which exist are either small (and not detectable by the data set) or larger, but they are either
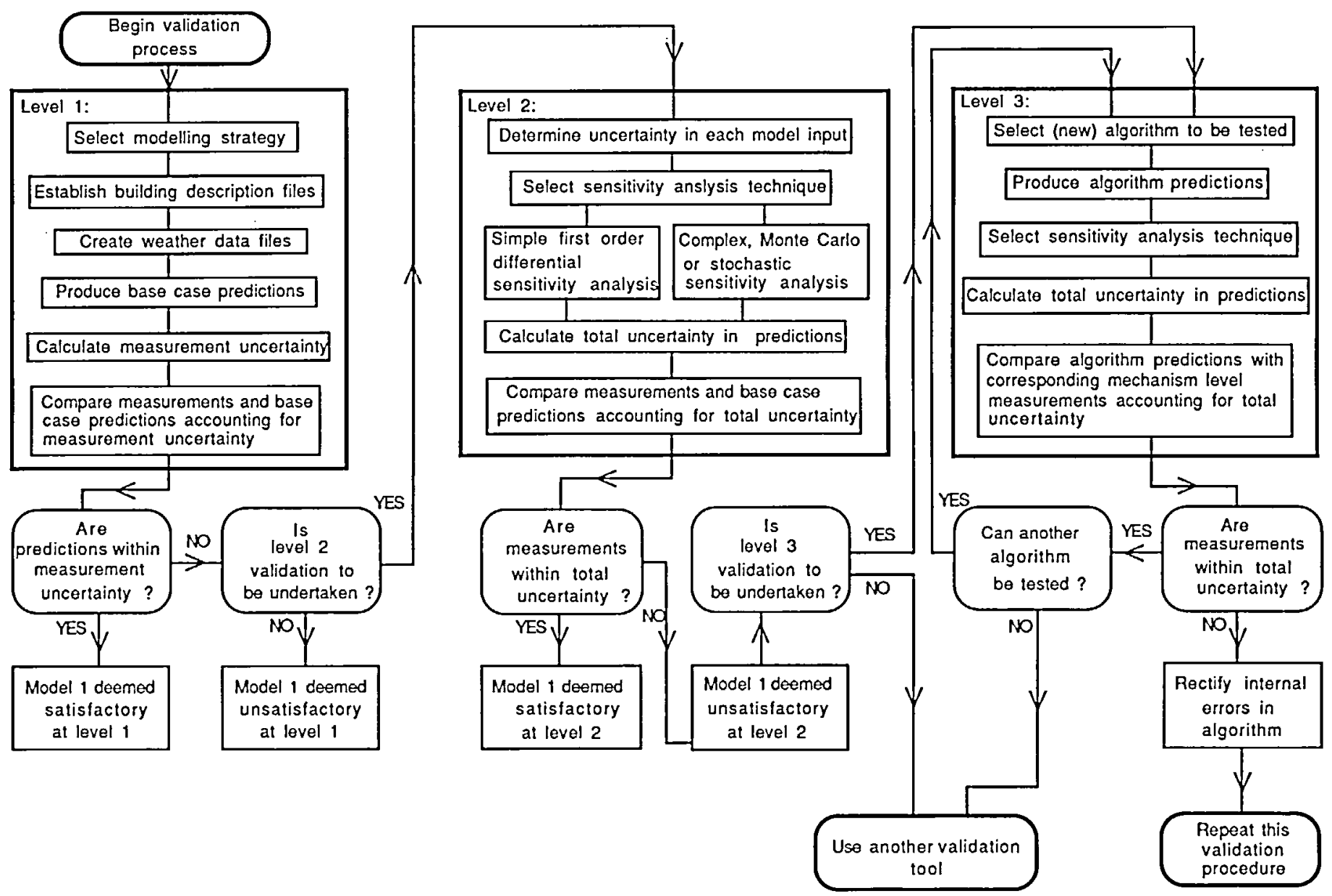

Figure 1 Three-level empirical validation methodology 


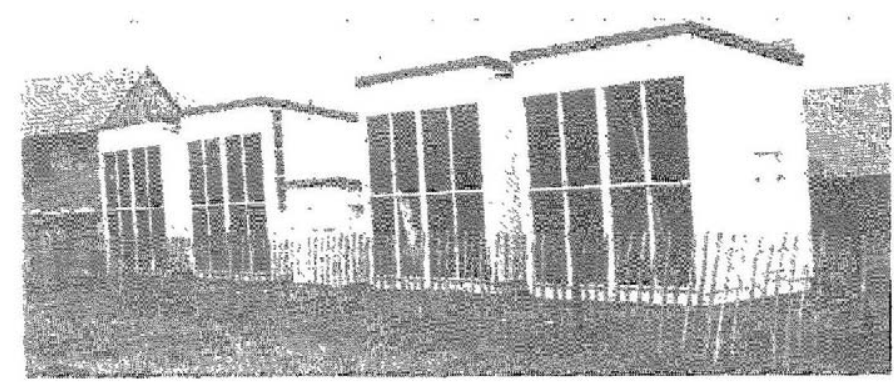

Figure 2 Test cells monitored by the Polytechnic of Central London, Peterborough, LK

compensated for by othes internal errors (which have an equal but opposite effect) or they lie in parts of the model which are not stressed by the data set chosen or the parameters compared.

The validation procedure has been deliberately developed so that manipulation of the computer code within the model itself is unnecessary. The method is therefore applicable to commercially available models, as well as those in the public domain, and as such it has advantages over some other validation techniques.

\section{Level I procedure}

\subsection{Modelling the PCL cells}

Using a Class A approach, a single base case prediction is made and there is no opportunity for subsequent refnements (e.g. if the model perfoms badly) so it is essential that the building is modelled as accurately as each model allows. Blunders in the input data (an unquantifable source of external error) must be avoided and all sources of uncertainty must be noted.

The exterior surfaces of the PCL cells were of weli insulated and well sealed stud frame construction with a stressed skin plywood facing. This outer shel was bolted together on site from prefabricared units built at the PCL. The area below a suspended ceiling was divided by a central partition into two cells of equal volume, $2.16 \mathrm{~m}$ deep by $1.6 \mathrm{~m}$ wide by $3.0 \mathrm{~m}$ high (Figures 2 and 3, Table 1).

The cells were modelled as an integral three-zone unit with all dimensions and constructiont detals being taken from the working drawings: ${ }^{(0)}$, supplemented by data gathered from research reports ${ }^{(11,12,13}$ and by visiting the site in Pererborough,

Because the cells had a wooden frame construction, each surface consisted of numerous crossusectional types (Figure 3, Table 1). To avoid introducing any approximations, each of these was modelled croticity at septrate sub-surface. In all, the cells consisted of 34 sut-surfaces and the roof space 28 sub-surfaces. Values given in the CIBSE Guide were used for all material thermophysical properties, cavity resistances, surface radiative properties and glazing transmission characteristics.

The exposed rural site ensured that these were no site objects shading the south facing windows. The tacade shading due

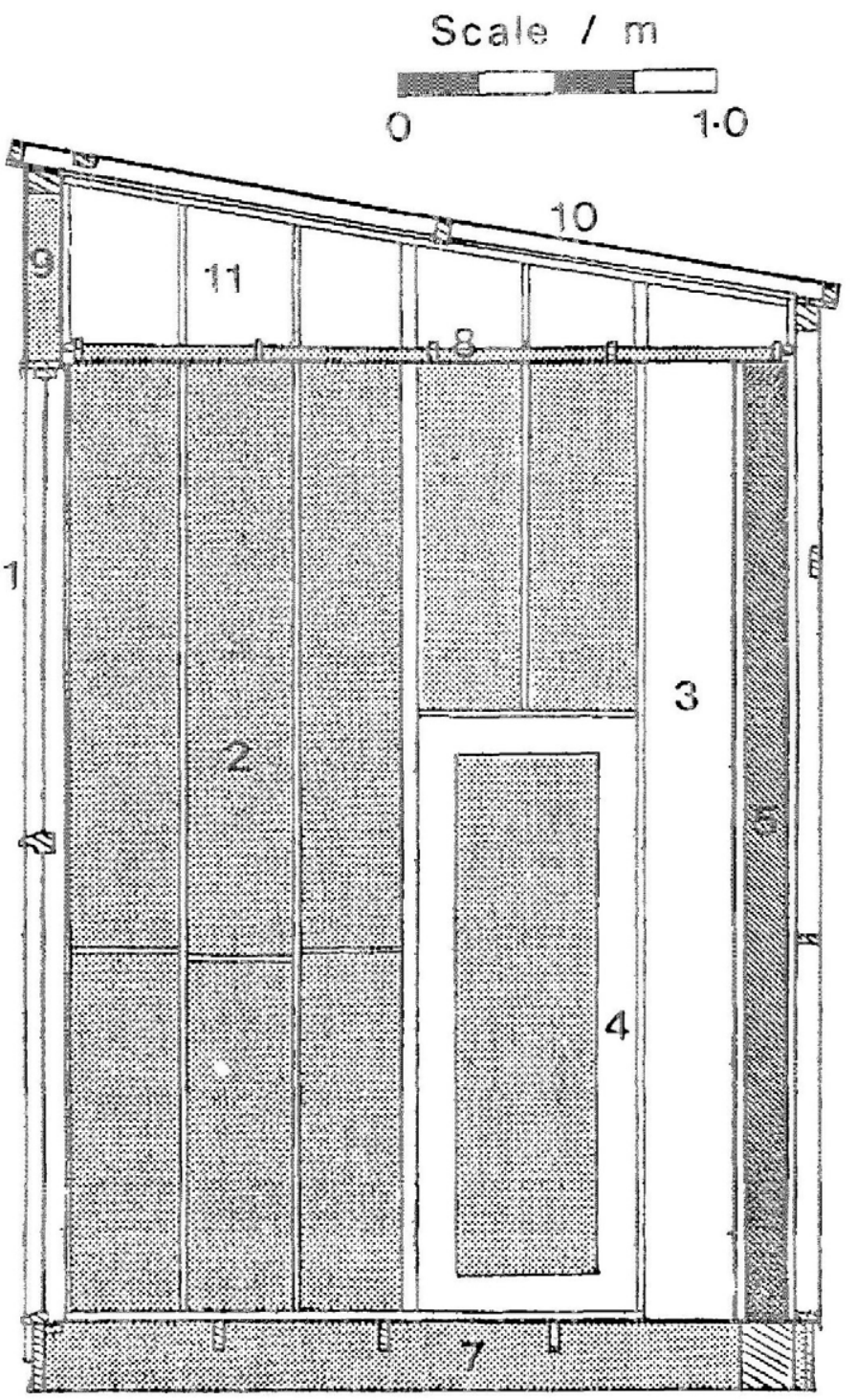

Figura 3 Construction of PC test ceils: Detailed cross-section of cell 2 looking west, showing franing members, door hatçs, insulation and masony wall

Table $\quad L^{\prime}$-valuest for major sub-stirfaces in Figure 3 as colculated by ESP

\begin{tabular}{|c|c|c|}
\hline $\begin{array}{l}\text { Key } \\
\text { Fig. 3) }\end{array}$ & Sub-suriace & $U\left(\mathrm{~W} \mathrm{~m}^{-2} \mathrm{~K}^{-1}\right)$ \\
\hline 1 & Windows & 5.6 \\
\hline 2 & Insulated side wal! & 0.7 \\
\hline 3 & Lrinsulated side wail & 59 \\
\hline 4 & Door frame & 1.9 \\
\hline 5 & Viasoncy wail & 3.2 \\
\hline 6 & Parly wa!l & 0.3 \\
\hline 7 & Fioor & 0.2 \\
\hline 3 & Cesting & 0.4 \\
\hline 9 & Berint & 0.4 \\
\hline 10 & Root & 3.7 \\
\hline 1 & Roof spece sides & 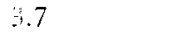 \\
\hline
\end{tabular}

- Al at of and strace resistances used are CIBSE values for verical interior valls: raminur menters are ignor:d. 
to the mullion and rail supporting the single glazing was modelled explicitly by all three models since preliminary sensitivity analysis indicated that cell air temperatures could be overpredicted by as much as $2 \mathrm{~K}$ if the shading was neglected. It should be noted here that a Class $A$ approach does not preclude such preliminary studies being undertaken, provided that no reference to the measured results is made. The shading of the side wall of cell 2 caused by the adjacent cell (Figure 2) was also modelled explicitly by ESP; however, it could not be modelled by SERIRES and HTB2. Sensitivity analyses suggested that this could lead to an overprediction in the peak cell air temperature of about $0.7 \mathrm{~K}$. This was the only feature of the cells which could not be treated in an identical and rigorous manner by the three programs.

The weather and building performance data collected on site were checked carefully and only three obviously spurious recordings were found. These were rectified. Computer programs were written to calculate the missing parameters required by the models, most significantly the direct normal irradiance required by SERIRES. Algortihms taken from the meteorological database handbook ${ }^{(14)}$ were used for all these manipulations.

The free-floating air temperature measured in cell 2 for the period 4-12 May 1984 will be used throughout this paper to illustrate the validation methodology (Figure 4), although assessments for other periods and for window and mass wall temperatures have also been successfully undertaken ${ }^{(2)}$.

At the start of each simulation, the models choose a different default initial node temperature and assume zero internodal heat flux, so a preconditioning period is necessary before reliable predictions can be obtained. The models also require the number of nodes to be used in each layer of material to be specified and ESP also requires the user to define the simulation time step. Preliminary sensitivity studies led to the adoption of 4 days preconditioning, 3 nodes per layer and 4 time steps per hour in ESP. The error in the cell 2 air temperatures due to the adoption of these values was estimated at less than $1 \mathrm{~K}$.

\subsection{Base-case predictions}

A direct visual assessment of the base-case predictions was obtained by plotting the predicted values against the measured values directly (Figure 5 top) and also by plotting them as the difference from the measured values (Figure 5 bottom). In addition, the following statistics, as proposed by Judkoff et al. ${ }^{(15)}$, were routinely calculated as numerical measures of the overall differences between the predicted
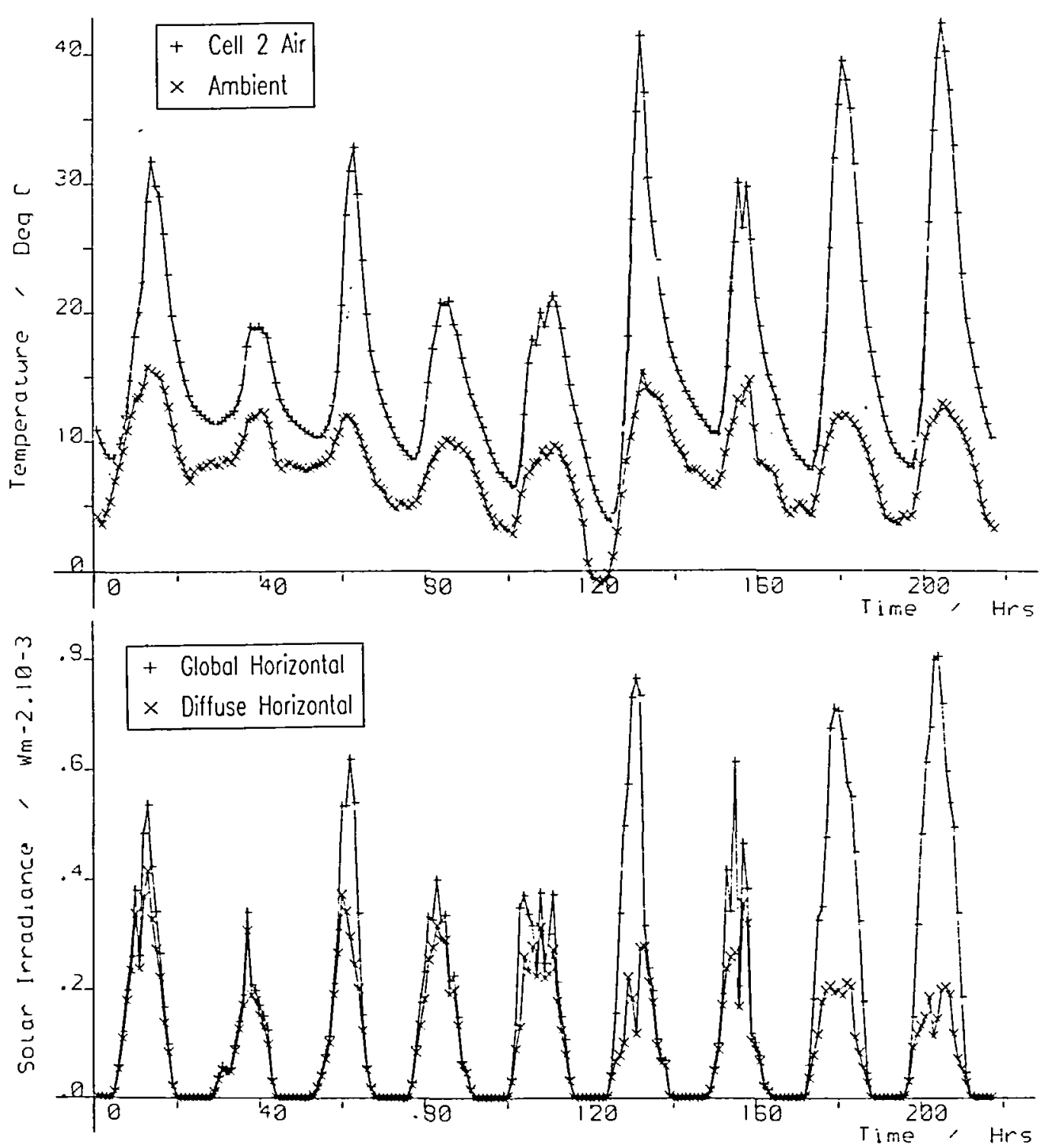

Figure 4 Measured weather data and cell 2 air temperature 

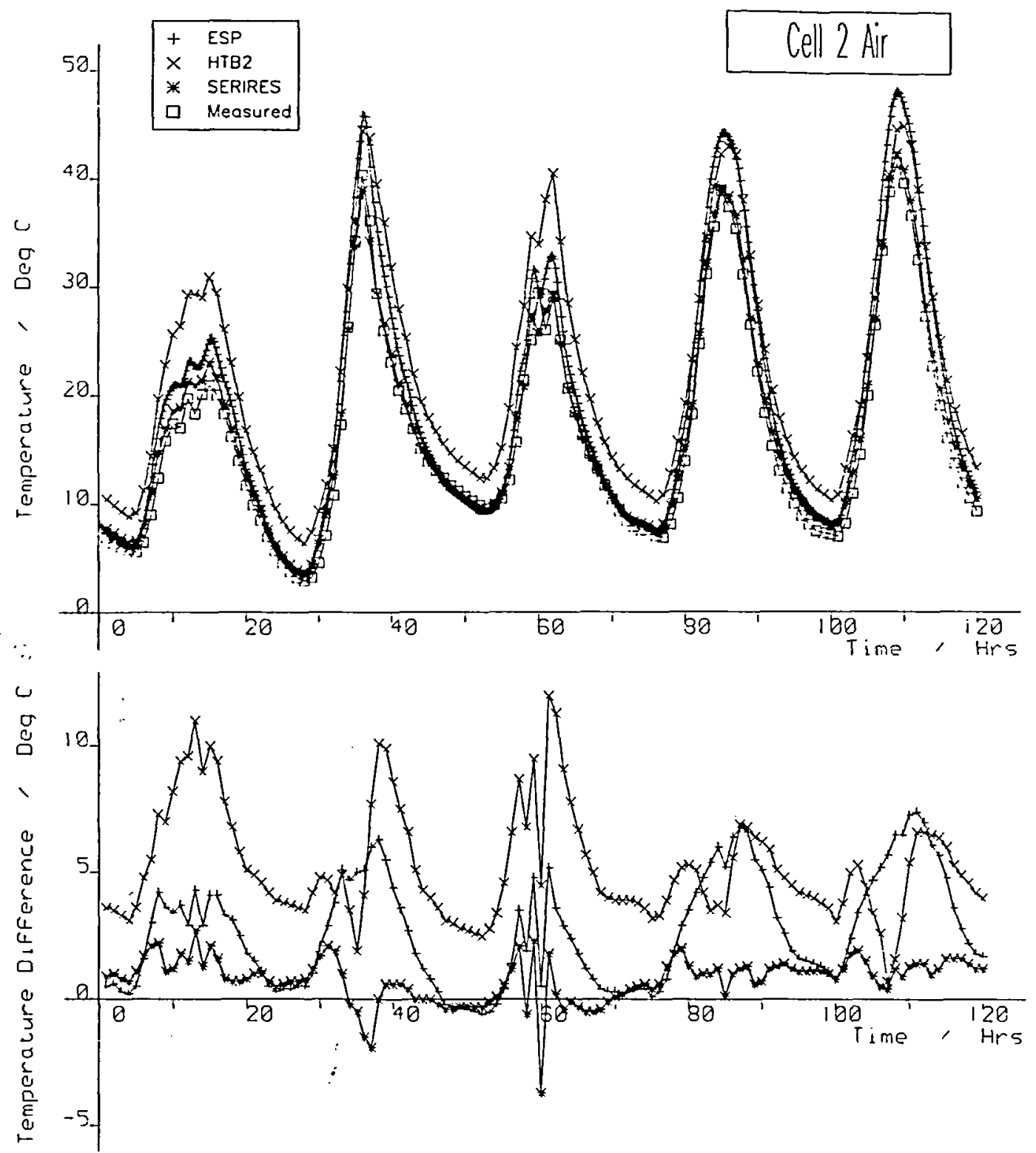

Figure 5 Comparison of measured $\left(T_{\mathrm{mu}}\right)$ and predicted $\left(T_{\mathrm{pr}}\right)$ cell temperatures and temperature differences $\left(D_{i}\right)$

and measured values (Table 2); definitions for Table 2 are given in Table 3.

In Table $3 T_{p t}$ is the predicted temperature at hour $t\left({ }^{\circ} \mathrm{C}\right)$, $T_{\mathrm{m} t}$ is the measured temperature at hour $t\left({ }^{\circ} \mathrm{C}\right), N$ is the total number of hours in the comparison period.

Cross-correlation analyses were also conducted to assess the phase relationships between the measured and predicted values but it is beyond the scope of this paper to discuss this aspect.
Overall, the enclosure temperature predicted by SERIRES was much closer to the measured air temperature (RMS difference $1.2 \mathrm{~K}$ ) than the temperatures predicted by either ESP (RMS difference $3.5 \mathrm{~K}$ ) or HTB2 (RMiS difference $5.7 \mathrm{~K}$ ). There was no evidence of systematic differences in the SERIRES results; however, the results from ESP and HTB2 diverged dramatically from the measured values during the daytime with peak differences of $7.4 \mathrm{~K}$ for ESP and $12.0 \mathrm{~K}$ for HTB2.

These differences are very large (internal comfort assessment

Table 2 Statistical comparison of measured and predicted air temperatures

\begin{tabular}{|c|c|c|c|c|c|c|c|c|}
\hline \multirow[t]{2}{*}{ Model } & \multicolumn{7}{|c|}{ Differences between measured and predicted temperatures } & \multirow{2}{*}{$\begin{array}{l}\text { Cross } \\
\text { correlation } \\
\text { at zero time } \\
\text { delay }\end{array}$} \\
\hline & $\begin{array}{l}\text { Maximum } \\
\text { temp. } \\
\left({ }^{\circ} \mathrm{C}\right)\end{array}$ & $\begin{array}{l}\text { Time } \\
\text { (h) }\end{array}$ & $\begin{array}{l}\text { Minimum } \\
\text { temp. } \\
\left({ }^{\circ} \mathrm{C}\right)\end{array}$ & $\begin{array}{l}\text { Time } \\
\text { (h) }\end{array}$ & $\begin{array}{l}\text { Mean } \\
\text { temp. } \\
\left({ }^{\circ} \mathrm{C}\right)\end{array}$ & $\begin{array}{l}\text { Absolute } \\
\text { mean } \\
\text { temp. } \\
\left({ }^{\circ} \mathrm{C}\right)\end{array}$ & $\begin{array}{l}\text { Root mean } \\
\text { square temp. } \\
\left({ }^{\circ} \mathrm{C}\right)\end{array}$ & \\
\hline ESP & 7.4 & 111 & -0.6 & 52 & 2.7 & 2.7 & 3.5 & 0.99 \\
\hline SERIRES & 2.7 & 13 & -3.7 & 60 & 0.8 & 1.0 & 1.2 & 1.00 \\
\hline HTB2 & 12.0 & 61 & 0.7 & 107 & 5.2 & 5.2 & 5.7 & 0.98 \\
\hline
\end{tabular}


Table 3 Definitions of quantities for Table 2

\begin{tabular}{lll}
\hline Difference & $D_{t}=T_{\mathrm{p} t}-T_{\mathrm{m} t}$ & $(1)$ \\
Largest difference & $\tilde{D}=\operatorname{Max} D_{t}$ & $(2)$ \\
Smallest difference & $\bar{D}=\operatorname{Min} D_{t}$ & $(3)$ \\
Mean difference & $\bar{D}=\Sigma\left(D_{t}\right) / N$ & $(4)$ \\
Absolute mean difference & $|\bar{D}|=\Sigma \mid D_{t} / / N$ & $(5)$ \\
Root mean square difference & $\left(D^{2}\right)^{1 / 2}=\left(\left(\bar{D}_{t}\right)^{2} / N\right)^{1 / 2}$ & $(6)$ \\
\hline
\end{tabular}

demands predictive accuracy to within about $\pm 1 \mathrm{~K}$ ); however, because the conditions in the cells were rather extreme, (diurnal temperature variations over $40 \mathrm{~K}$ ) the impact of any internal and external errors is likely to be exaggerated. The true significance of these differences can, however, only be assessed by an analysis of errors.

\subsection{Estimating the measurement uncertainty}

Typically, measurement errors have a fixed component (due to the intrinsic accuracy of the sensor, the recording technique and the method of mounting the sensor) and a variable component (in the case of cell air temperatures, due to stratification of air in the cells). For the air temperature sensor in the PCL cells, the error in the sensing and recording technique was estimated as $\pm 0.3 \mathrm{~K}^{(10)}$ but an additional component of $\pm 0.2 \mathrm{~K}$ was added since, despite careful shielding, the sensor could be influenced by radiant heat exchange. The degree of stratification in the PCL cells was a function of the difference between the ambient and internal air temperature. The maximum floor-to-ceiling variation was estimated to be $10 \mathrm{~K}$ when the inside-to-outside temperature difference was greatest $(29.7 \mathrm{~K}$ at $1300 \mathrm{~h}$ on $12 \mathrm{May})$. The centrally located air temperature sensor would, however, give a reasonable measure of the spatial average (which is the parameter predicted by the models) so the variable error component was taken to be half the floor-to-ceiling temperature variation. The total measurement error was therefore taken to be:

$$
e_{\mathrm{m} t}= \pm\left[0.5+0.084\left(T_{\mathrm{m} t}-T_{\mathrm{at}}\right)\right]\left({ }^{\circ} \mathrm{C}\right)
$$

where $T_{a t}$ is the measured ambient temperature at hour $t$ $\left({ }^{\circ} \mathrm{C}\right)$ and $T_{\mathrm{m}}$ is the measured cell air temperature at hour $t$ $\left({ }^{\circ} \mathrm{C}\right)$.

The error band generated by this equation (Figure 6) appears rather large considering the rigour with which the experiments were undertaken. However, the band boundaries have been estimated such that there is only a small, say $1 \%$, chance that differences between the actual and measured air temperatures would lie outside the upper boundary and only $1 \%$ chance that this difference would be below the lower boundary. Thus, if hourly predictions lie outside these boundaries more frequently than once every 100 hours it is very unlikely that they are a true reflection of the actual air temperature.

\subsection{Level 1 assessment of models}

It can readily be seen (by comparing Figures 5 (bottom) and 6) that the temperature differences $D_{t}$ exceed the measurement error $e_{\mathrm{m} t}$ virtually all the time for models ESP and HTB2; clearly, these models are not valid at Level 1. By plotting $D_{t}$ against $e_{\mathrm{m} t}$ for SERIRES (not shown here) it is apparent that the SERIRES predictions exceed the upper boundary of the measurement uncertainty approximately $40 \%$ of the time; well in excess of the $1 \%$ occurrence rate to be expected by chance. Thus, SERIRES is also invalid at Level 1.

The errors in the predictions of the three models could be due to internal errors in the code or external errors in the input files; the Level 2 procedure seeks to clarify which of those two alternatives is the cause.

\section{Level 2 procedure}

\subsection{Alternative sensitivity analysis techniques}

Because thermal models are complex, there is no simple (analytical) way of determining the uncertainty in the predictions due to the uncertainty in the inputs. Therefore, all the input errors must be propagated (numerically) through

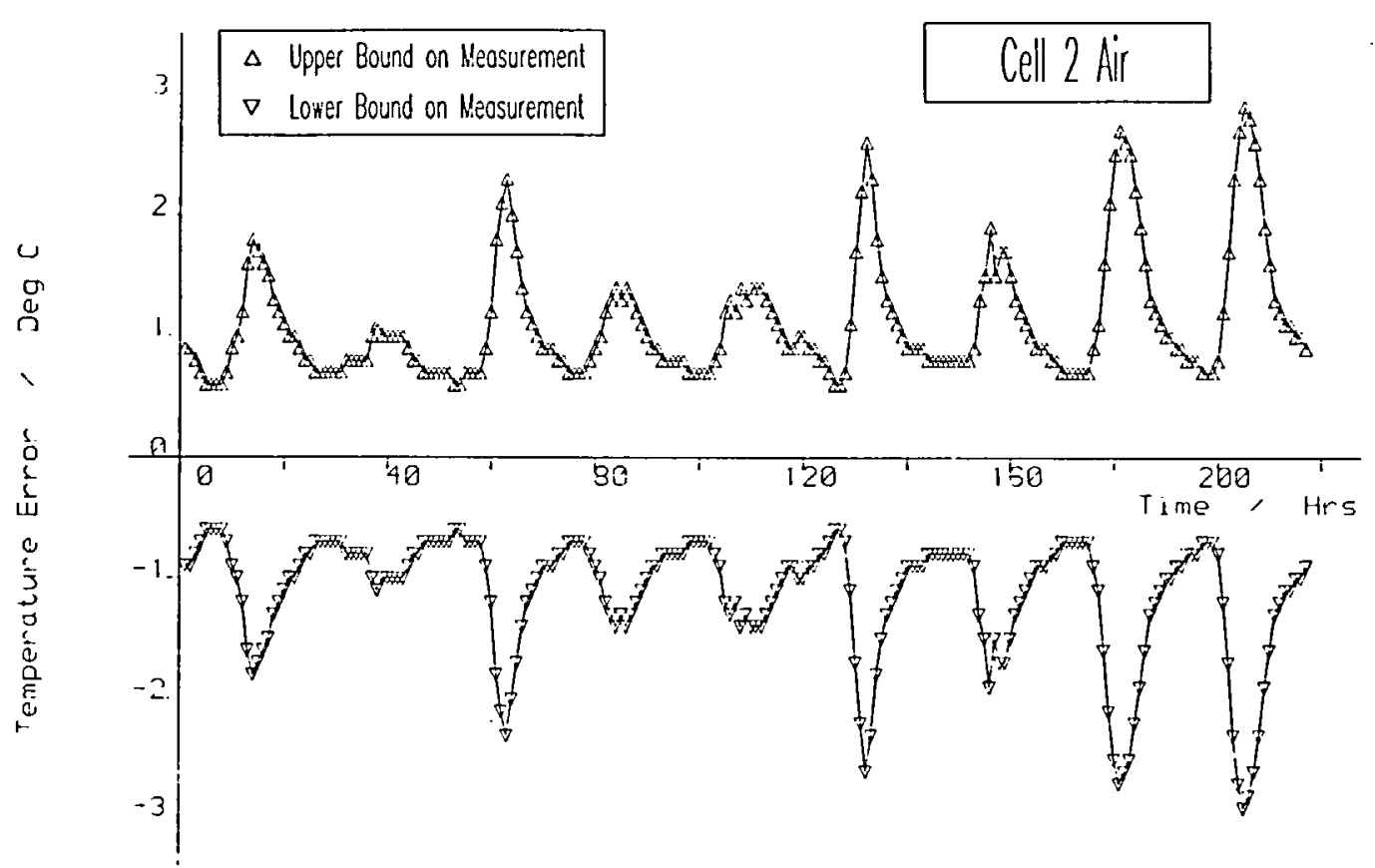

Figure 6 Uncertainty in measured cell air temperatures $e_{\mathrm{m} t}$ 
the model to establish the total uncertainty in the outputs. There are two widely recognised techniques for doing this: the Monte Carlo method and the simple (first-order) differential sensitivity method. Less well established, in the building thermal modelling field at least, is the stochastic sensitivity technique. These three techniques are being evaluated at Leicester Polytechnic but it is clear that both the stochastic technique and, to a lesser extent, the Monte Carlo method, are computationally complex, and so unlikely to prove suitable for a widely applicable validation tool. Initially, therefore, Level 2 validation was based on simple differential sensitivity analysis.

This technique involves perturbing each input parameter in turn, within the estimated error bounds, to determine the individual parameter sensitivities. Then, the predicted sensitivities are added in quadrature to determine the total error band around the predictions. To demonstrate the technique the analysis is illustrated for ESP and SERIRES and results are shown for one typical day, 12 May.

The principal disadvantage of this method is that it is only strictly valid if the errors in each input are linear and independent in their effect. Preliminary studies were therefore undertaken ${ }^{(2)}$ to establish that this requirement was substantially upheld for the particular models (ESP, SERIRES) and predicted parameter (air temperature in cell 2) in question. These studies revealed that the error band width and shape were correctly calculated, although the bounds defining the total uncertainty may be overestimated by about $5 \%$. The differential analysis technique was therefore deemed acceptable in this particular case, but the error in the predicted error bounds was taken into account when evaluating the models. Others have also noted that sensitivities tend to be approximately linear ${ }^{(16)}$.

It is important to check that the linearity and independence restriction is substantially upheld whenever differential sensitivity analysis is adopted. Further work is underway at the Polytechnic to evaluate the reliability of the method by comparing the technique with those based on stochastic sensitivity and the Monte Carlo approach. The techniques are being compared using continuously and intermittently heated domestic-scale buildings.

\subsection{Input parameter uncertainty}

A through literature search was undertaken to provide estimates of the upper and lower bound values for each model input parameter such that there was only $1 \%$ likelihood that the PCL cells, as erected on site, had values outside these bounds. Clearly, while the literature review can provide the basis for selection, a degree of estimation is involved; in all cases of doubt the error bounds were overestimated. These error bounds and the basis of calculation are likely to be of value to others wishing to undertake sensitivity analysis ${ }^{(2)}$.

The upper-and lower-bound values were often asymmetric about the base-case values. For example, the extinction coefficient for a perfectly clean window was assumed for the base-case value; this value is unlikely to get smaller (except due to variations between glass sheets) but it could get much larger due to dirt films. Thus, a maximum value exists whereas, to the accuracy quoted, there is no minimum value.

The critical combinations of thermophysical properties (i.e. those which produced the greatest increase and the greatest decrease in peak air temperature) were determined by preliminary sensitivity analyses. In general, the greatest temperature increase occurred when the density, conductivity and specific heat capacity took their smallest likely values (i.e. materials had minimum thermal mass and maximum thermal resistance) and the greatest temperature decrease occurred when the three parameters took their maximum likely values; the only exceptions were for the timber framing and fibreglass insulation.

Some parameters frequently required by the models were not included in the analysis, in particular, site location and internal cell geometry. This is because both were known very accurately, and any uncertainty associated with them was likely to be encompassed either by the uncertainty in the thermophysical properties and surface coefficients (for cell geometry) or by the weather data and ground reflection (for site location).

To account for the uncertainty in the weather data, a number of weather data files were established for each model. Each file contained the base-case data except for one parameter, which was replaced by either the maximum or minimum values calculated for each hour.

\subsection{Individual sensitivities}

To determine the individual sensitivities, ESP and SERIRES were each run many times with one input parameter varying while the other parameters remain fixed at their base-case values. In general, each input parameter had a maximum and a minimum value which had to be used so, for ESP for example, since there were 28 uncertain inputs around 56 simulations were needed.

At any hour $t$, and for any parameter of interest $i$, there is a difference in the hourly predictions as a result of changing the input value to either the maximum or minimum value (e.g. Figure 7 (top)). The error at each hour due to adopting the maximum value can be expressed as

$$
\hat{e}_{i t}=\hat{T}_{i t}-T_{p l}\left({ }^{\circ} \mathrm{C}\right)
$$

where $\hat{T}_{i t}$ is the predicted temperature at hour $t$ using the maximum value of parameter $i\left({ }^{\circ} \mathrm{C}\right)$ and $T_{p t}$ is the predicted temperature at hour $t$ using base-case inputs $\left({ }^{\circ} \mathrm{C}\right)$. Similarly, there is an error when the input parameter adopts the minimum value:

$$
\check{e}_{i t}=\check{T}_{i t}-T_{p t}\left({ }^{\circ} \mathrm{C}\right)
$$

where $\check{T}_{i t}$ is the predicted temperature at hour $t$ using the minimum value of parameter $i\left({ }^{\circ} \mathrm{C}\right)$.

Since the maximum and minimum values of each parameter tend to be respectively higher than, and lower than, the base-case values, the two errors at each hour, $\hat{e}_{i t}$ and $\breve{e}_{i t}$, tended to have opposite signs (Figure 7 (bottom)). Furthermore, because the base case value of the parameter was not always exactly midway between the maximum and minimum values, the two errors tended to have different magnitudes (Figure 7 (bottom)). In a few cases, however, particularly when alternative rather than maximum or minimum parameter values are used, such as for internal solar distribution, the two errors may have the same sign at certain times.

The sensitivity of the peak air temperature predicted by ESP and SERIRES to the uncertainty in the individual model inputs was obtained (Figure 8) and in a similar way so was the sensitivity of the overall temperature predictions as expressed by the root mean square change over the day (not illustrated). In either case, the sensitivities to the input parameters showed a similar ranking. 

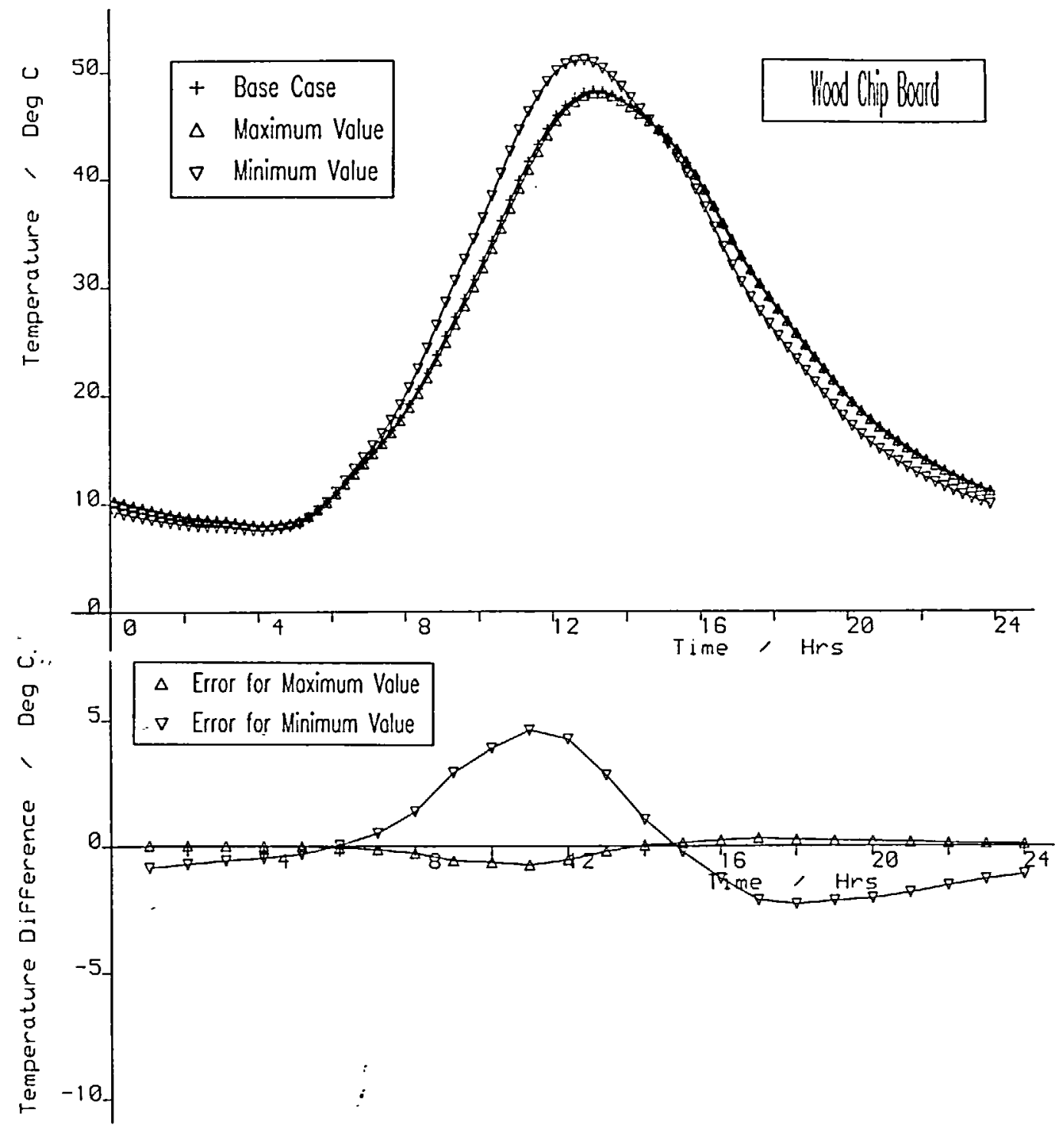

Figure 7 Error in ESP cell temperature predictions due to uncertainty in thermophysical properties

Contributing the greatest uncertainties were: $(a)$ the external surface coefficients used by SERIRES; $(b)$ the window $U$ values; $(c)$ the solar lost coefficients used by SERIRES and $(d)$ the ground reflectivity. Interestingly, although the weather parameters were carefully recorded, the tiny uncertainties in the data led, in general, to uncertainty in the peak air temperature of similar magnitude to the uncertainty induced by very large uncertainties in the thermophysical properties data.

Simple sensitivity analysis has a valuable role to play when designing experiments for model validation because it can show which parameters must be measured accurately in order to minimise uncertainty. It can thus help to produce data sets capable of discriminating between valid and nonvalid models at a very fine level.

\subsection{Total uncertainty}

Once all the simulations had been completed and the result files stored, the total positive error at any given hour was calculated by adding in quadrature all the errors with a positive sign at that hour (either $\hat{e}_{i t}$ or $\check{e}_{i t}$ ). Similarly, the total negative error at any hour was calculated by adding in quadrature all the errors with a negative sign. These calculations were repeated for each hour in turn.
In cases where the two errors had the same sign (e.g. both positive), the largest absolute magnitude was used when calculating the positive total error and zero was used in the calculation for the total negative error. (The same procedure, but reversing the signs, was used if both $\hat{e}_{i t}$ and $\check{e}_{i t}$ had negative signs).

It was also necessary to take into account the uncertainty in the measurements and the most effective way of doing this was to include the measurement uncertainty in the quadrature addition calculation; this method has been recommended by others ${ }^{(17)}$.

Since the input and measurement errors were defined so that there was only about $1 \%$ probability of their being exceeded, the total positive error at any hour represents the 99 percentile error bound and is given by:

$$
e_{99 t}= \pm\left(e^{2}{ }_{a t}+e^{2}{ }_{b t} \ldots+e^{2}{ }_{i t} \ldots+e_{n t}^{2}+e_{m t}^{2}\right)^{1 / 2}
$$

where $e_{i t}>0$ for all $i$ and $e_{m t}>0$.

Similarly the total negative error represents the onepercentile error bound and is given by

$$
e_{i t}= \pm\left(e_{a t}^{2}+e_{b t}^{2} \ldots+e^{2}{ }_{i t} \ldots+e_{n t}^{2}+e^{2}{ }_{m}\right)^{1 / 2}
$$

where $e_{i t}<0$ for all $i$ and $e_{m t}<0$. 


\begin{tabular}{|l|l|}
\hline \multicolumn{1}{|c|}{ Input Parameter } \\
\hline Ground Reflectivity \\
Index of Exposure \\
Orientation
\end{tabular}

${ }^{1}$ Input parameler not requested by ESP. ${ }^{2}$ Input parameter not requested by SERI-RES

${ }^{3}$ Thermophysical properties of given material

Figure 8 Sensitivity of predicted air temperature to uncertainty in model input parameters

A simple computer program was produced to access each pair of results files in turn, calculated the hourly errors $\left(\hat{e}_{i t}, \check{e}_{i t}\right)$ and add the squared hourly error terms into the appropriate (one or 99 percentile) calculation. The contribution of the measurement uncertainty was then included and the square root taken to give the hourly values of each error bound. Finally, the absolute values of the upper 99 percentile bound, $U_{t}$, and the lower 1 percentile bound, $L_{t}$, were calculated, where:

$$
U_{t}=T_{p t}+e_{99 t}
$$

and

$$
L_{t}=T_{p t}-e_{1 t}
$$

\subsection{Level 2 assessment of models}

The total uncertainty in the predictions (the differences between $U_{t}$ and $L_{t}$ ) was greatest around the time of the peak air temperature and was $11.3 \mathrm{~K}$ for ESP (Figure 9 (top)) and $12.3 \mathrm{~K}$ for SERIRES (Figure 9 (bottom)). The three models also produced similar values for the total uncertainty at all other hours.

The error band around the SERIRES predictions encompassed the measured values for all but two of the 24 hours (Figure 9 (top)) and for these two hours the measurements were only marginally outside the lower bound. The error bounds around ESP, however, while of similar magnitude to those around SERIRES, failed to encompass the measurements between 0500 and $2000 \mathrm{~h}$; the measurements also lay further outside the lower bound than for SERIRES (Figure 9).

Were the lower bound precisely positioned, these results would lead to the conclusion that internal errors exist within the models. However, it was noted above that the lower and upper bounds may be overpredicted by up to $5 \%$. If this were so then the measurements would always lie inside the error bounds for SERIRES but for most of the daytime the ESP predictions would still be too high.

It was concluded, therefore, that there was insufficient evidence to indicate the presence of internal errors in SERIRES (and that the divergent predictions could be accounted for by the uncertainty in the measurements and model input data). SERIRES was therefore deemed to be valid at Level 2 for the prediction of air temperature in PCL cell 2 on 12 May; note the restrictions placed on the conclusions. For ESP, however, it was concluded that internal errors may exist in the model and that it was not valid at Level 2 for the particular conditions under test. The nature of these internal errors is investigated at Level 3.

\section{Level 3 assessment}

Having identified the likely presence of internal errors in ESP, the third level of the validation methodology seeks to identify the nature of these errors. The following sources of error are possible for the PCL cells:

(a) the use of a $U$-value rather than a transparent multilayer construction for modelling the window;

(b) the use of fixed, or variable but incorrect, internal and external surface convection coefficients;

(c) the use of an inappropriate, anisotropic sky model; or

(d) failure to account for the solar radiation reflected back through the windows.

Of these alternatives, it was only possible to investigate $(c)$ using the PCL data alone since the south-facing vertical irradiances were the only mechanism level parameters measured in the cells.

The analysis indicated that ESP predicted higher incident radiation values than the other two models, both of which used an isotropic sky model (Figure 10). The difference on 12 May was about $90 \mathrm{~W} \mathrm{~m}^{-2}$ which, using the model sensitivity to global solar radiation (Figure 8), suggests an internal air temperature prediction by ESP about $5.6 \mathrm{~K}$ above that for the other models; this is close to the observed difference in prediction at $1300 \mathrm{~h}$ of $5.5 \mathrm{~K}$. This seems a reasonable explanation of the ESP predictions. However, it was in fact ESP, rather than the SERIRES and HTB2, which predicted irradiances closest to the measured values (Figure 10). The indication is that the SERIRES and HTB2 isotropic sky models are inadequate but, at least for the PCL cells, this is being compensated for by errors in other parts of the model. 

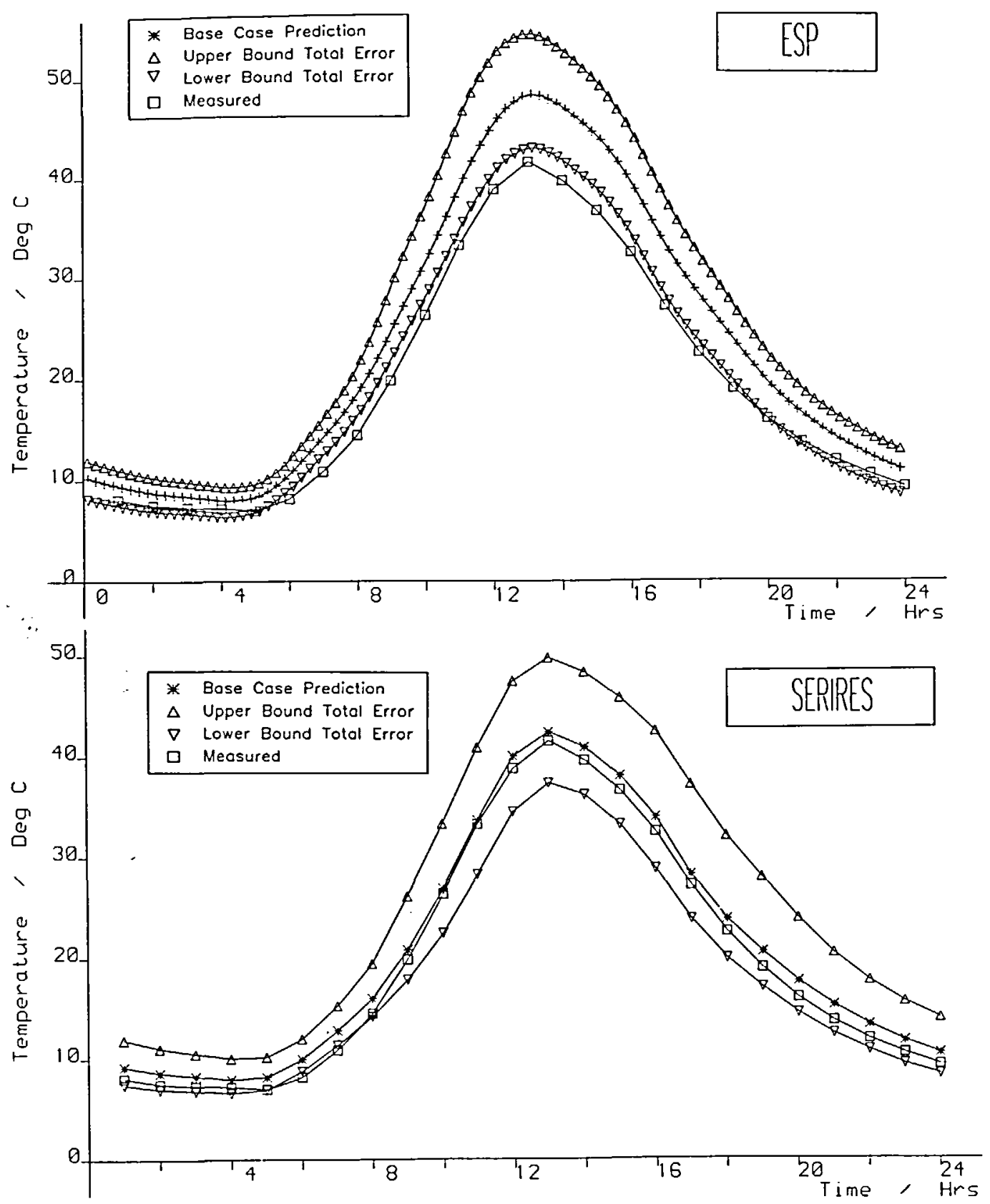

Figure 9 Comparison of cell temperatures predicted by SERIRES and ESP with measured values showing total error bands around predictions

Unfortunately, because no other mechanism level was available, it was not possible to pursue the investigation further using measured data for the PCL cells alone. Measured data from other test facilities, or the use of other validation techniques, such as analytical tests or intermodel comparisons, must therefore be used to isolate the internal errors in ESP. For example, to investigate the possibility of errors in the window conduction algorithms in ESP, the predictions of the algorithm could be compared directly with those from dedicated window models, such as VISION ${ }^{(18)}$ or WINDOW $2.0^{(19)}$; alternatively measured mechanism level data could be obtained from a window test facility, such as the NBS Passive Solar Calorimeter ${ }^{(20)}$ or the MOWITT facility $^{(21)}$. The interaction of empirical validation with other validation techniques is discussed below.

\section{An empirical validation tool}

One of the striking results of the review of data sets ${ }^{(3)}$ was the poor level of documentation and in particular the lack of a site handbook describing the test facilities and the data collected. Notable exceptions are site handbooks produced by the NIST ${ }^{(22)}$ and the SERI ${ }^{(23,24)}$. A site handbook for the PCL data set has therefore been produced which, it is hoped, will act as an exemplar for the documentation of future data sets.

The hourly weather data and the measured air, window and mass wall temperatures for two periods (25 February to 4 March and 4 May to 12 May) have been simplified, reformatted, and transferred onto an IBM compatible floppy disk. In addition a 'user guide' has been produced which explains how to use the handbook and data disk effectively to validate a thermal model. The advice is based on the methodology outlined in this paper.

\section{Reflections on the methodology}

It was noted above that validation often uses a Class B approach in which input parameters are varied, within the bounds of plausibility (the error bounds), to improve the fit 


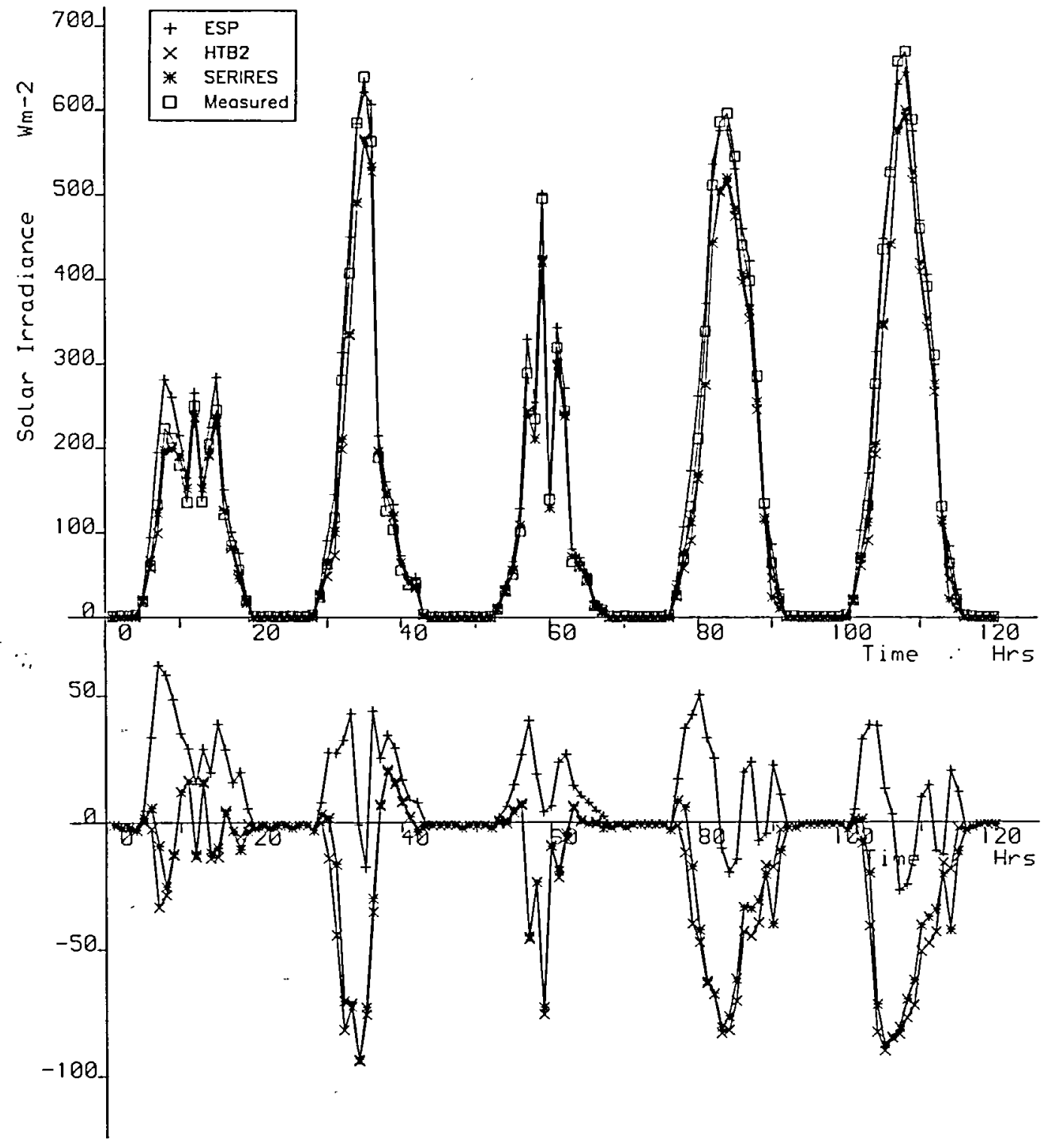

Figure 10 Comparison of measured and predicted south facing vertical irradiances and differences between predicted and measured values between measurements and predictions and, provided a good fit is ultimately achieved, the model is said to be valid. For the PCL cells, the scope for manoeuvring the predicted results was estimated using ESP by selecting various input combinations, making sure that each input was selected from within its estimated range of uncertainty. The highest and lowest temperature profiles predicted for 12 May were such that the peak temperature could be made to lie anywhere between $60^{\circ} \mathrm{C}$ and $35^{\circ} \mathrm{C}$. Thus the measured results (Figure 9 (top)) could easily be matched by numerous (hundreds) of different combinations of plausible input values.

It may be concluded therefore, that even with high-quality data (such as the PCL data) the scope for manoeuvring a good fit between measurements and predictions is so large that the Class B method is extremely unlikely to reveal the existence of internal errors in models. Thus, models may be accepted as valid even if they contain serious errors. Claims about model accuracy which are based on a Class B validation strategy should be treated with scepticism. The Class A approach, which is based on a thorough statistical assessment of errors, is a far more discriminating method and is thus likely to give a more accurate assessment of a model validity.

\section{Strategic use of validation tools}

Attempts have been made to monitor structures in such a way that they permit the assessment of building level predictions (e.g. energy inputs, air temperatures) as well as the accuracy of individual algorithms $s^{(15,23,24)}$. However, such data collection is extremely expensive and time consuming and the resulting data set becomes large and unmanageable. It is therefore recognised that a complete understanding of the sphere of validity of any thermal model is unlikely to be achieved using a single data set or indeed a single validation technique. An overall validation strategy is needed which employs a number of validation techniques in order to take full advantage of their strengths and to avoid the inconveniences of their weaknesses.

The methodology for empirical validation proposed here is believed to be sound, although the exact sensitivity analysis techniques could be changed. Despite deliberate attempts to keep the method simple (by not requiring coding modifications, or advanced mathematical expertise) a significant commitment both in terms of time and computer resources is still needed. In addition, skilled experimenters are needed 
to configure and monitor suitable test buildings. (The failure of extant data sets to meet even the most limited of acceptance criteria for empirical validation ${ }^{(3)}$ is indicative of the difficulty of this task.) It is likely, therefore, that empirical validation tools will be employed by a limited number of people, using a limited number of data sets and a limited number of models. Given this, and the experience with the PCL data set, which illustrates that under a given set of conditions some models will be invalid (while others will appear to be valid), model validation is more likely to succeed using the following approach.

(a) A small number of empirical validation tools must be carefully chosen to cover the range of building types, modes of occupancy and weather conditions encountered in a particular geographical location. Each of these represents a 'validation node'.

(b) A small number of highly sophisticated models, such as those discussed in this paper, must be evaluated at each node. Any model which produces accurate predictions will be established as a 'benchmark model' for the given node. There may be more than one benchmark model for some nodes and for others, given the current state of the art, there may be none. It is unlikely that a single model will form the benchmark for all the validation nodes: Using analytical tests and mechanism level data, new models and algorithms can be developed (or existing models improved) so that they become accepted as benchmark models for particular nodes.

(c) To cover situations not represented by the validation nodes, the conditions represented by a chosen node would be incrementally changed and the predictions of the benchmark models for that node could be compared for this new situation. If they agreed to within a defined level of accuracy, the new conditions would form a 'standard verification test'. Ultimately, by repeatedly changing the conditions in a carefully chosen manner, the conditions will resemble those of the adjacent validation node. If the benchmark models continued to give comparable predictions, then a series of standard verification tests (based on intermodel comparisons) will have been established linking the nodes. (If conditions become such that the benchmark models begin to diverge, an additional empirical validation node, would have to be established, or analytical investigations undertaken, to improve predictive ability in this region).

Using the above strategy, it is in theory possible to establish a multi-dimensional space lattice covering the complete region in which the models will operate. The lattice will consist of a network of validation nodes (based on empirical validation) linked by standard verification tests (based on intermodel comparisons). For each node and each test case a limited number of benchmark models will exist.

Any other model can be validated for any set of conditions by comparing its predictions with those of the benchmark model appropriate to the conditions of interest. The uncertainty in the benchmark models' predictions must be known for each node and test case, and provided the new model is within this uncertainty bound it will be deemed to be valid. The bulk of the validation testing can therefore be based on intermodel comparisons which, compared with the other techniques, are relatively quick and simple to undertake. The major UK model assessment programme, Applicability Study 1 , being undertaken by Leicester Polytechnic, is based on this strategy ${ }^{(25)}$.
The management of a validation process such as this is a political decision. One possible scenario is that model developers submit to a standards office the predictions from their codes and a description of the conditions under which they were obtained. Here, the appropriate simulations would be conducted with the appropriate benchmark model and the results compared. Some form of validation certification could then be issued, with a statement which carefully defines the region over which the model is applicable. Mechanisms would have to be devised, and backed by legislation, to prevent certificates of validity being used fraudulently to claim validity of other codes, the same code after modification, or the same code under different sets of conditions. Such issues are well beyond the scope of this paper.

\section{Conclusions}

\section{1}

An empirical validation methodology has been developed and termed the Class A approach. Model predictions are made in ignorance of the actual measured building performance and uncertainties in the measurements and model data are accounted for statistically in a logical and systematic way.

\section{2}

The Class B approach, in which model inputs are varied systematically to obtain predictions comparable to the measurements, is extremely unlikely to reveal the existence of internal errors in models. Claims about model accuracy based on a Class B validation strategy should be treated with scepticism.

\section{3}

An empirical validation tool has been produced for the PCL test cells. It is presented as three items: a site handbook, a data disk, and a user's guide. It is able to assess the accuracy with which models predict air, mass wall and window temperatures in highly glazed, well insulated, well sealed, lightweight zones, with no mechanical heating or cooling. The validation tool stresses the accuracy of algorithms which predict conductive heat loss and solar gain through windows.

\section{4}

The errors in the air temperatures predicted by ESP could not be explained by the inaccuracy of the field measurements, the uncertainty in any one input parameter, or the total uncertainty due to all sources of external error. Therefore, it is highly likely that there were internal errors within ESP.

\section{5}

HTB2 produced the least accurate air temperature predictions. The values were well in excess of the estimated measurement uncertainty during both the day and the night. Although sensitivity analyses were not undertaken, it is unlikely that the errors could be explained by the total uncertainty in the validation process; they are more likely to be due to internal errors in the model.

\section{6}

No internal errors could be detected in SERIRES; it was deemed to be valid at Level 2 . 


\section{7}

Simple sensitivity analysis has a valuable role to play in the design of experiments to produce data for model validation since it can show which measurements are critical for minimising external errors. By reducing external errors the ability of the data set to identify the presence of internal errors is enhanced.

\section{8}

There is a need for more data sets on which to base empirical validation tools. These should be chosen to cover the range of building types, modes of operation and climatic conditions which the models are likely to be called upon to simulate. By measuring key model inputs these validation tools could have much greater resolution than the tool based on the PCL cells.

\section{9}

Empirical validation requires a considerable commitment of both time and resources. It is unlikely, therefore, that all - models will be tested using empirical validation tools. It is more likely that the tools will be used to validate a few benchmark models and to define their range of applicability. Simpler models will then be validated against the benchmark model which is appropriate to the conditions of interest.

\section{Acknowledgements}

My thanks are due to my colleague at Leicester Polytechnic, Prof. Neil Bowman, for his invaluable advice and support; the researchers at the collaborating institutions, the Building Research Establishment, Nottingham University and the Rutherford Appleton Laboratory, with whom I had numerous stimulating discussions; and the Science and Engineering Research Council for funding this work.

\section{References}

1 Bloomfield D Appraisal techniques for Methods of Calculating the Thermal Performance of Buildings Building Serv. Eng. Res. Technol. 6(1) 13-20 (1985)

2 Lomas K J and Bowman N T An Investigation into Analytical and Empirical Validation Techniques for Dynamic Thernal Models of Buildings Final Report to Science and Engineering Research Council on Grant No. GR/C/62871 Vol. 4 (1987)

3 Lomas K J A Compilation and Evaluation of Building Thermal Performance Data: With Special Reference to the Validation of Dynamic Thermal Models Building Energy Performance Assessment Club Report TN 90/4 (1990)
4 Cyber BLAST 3.0 User Information Manual (Alexandria, VA, USA: Control Data Corporation) (1980)

5 Higgs F S et al. DEROB-NBRI 2.0 (Three volumes) (Pretoria, S Africa: National Building Research Institute) (1983)

6 Clarke J A ESP Manual ABACUS (Glasgow: University of Strathclyde) (1982)

7 Palmiter $\mathrm{L}$ and Wheeling $\mathrm{T}$ SERI residential energy simulator verison 1.0 (Golden, CO, USA: Solar Energy Research Institute) (1983)

8 Bowman N T and Lomas K J Empirical validation of dynamic thermal models of buildings Building Serv. Eng. Res. Technol. 6(4) 153-162 (1985)

9 Alexander D K and Lewis P T HTB2-A Model for the Thermal Environment of Buildings in operation Technical Reference Manual rev. 0.0 (draft) (Cardiff: Welsh School of Architecture, UWIST) (1984)

10 Watson D M J Working drawings for the PCL Test Cells (Private Comm.) (1985)

11 Watson D M J and Littler J G F External Test Rooms Final Science and Engineering Research Council Report on grant GR/B/26626(London: Polytechnic of Central London) (1983)

12 Littler J G F, Watson D M J and Martin C J No-Fines Versus Concrete Blocks for Passive Solar Thermal Storage Report to Department of Energy via ETSU (London: Polytechnic of Central London Research in Building Group) RIB/84/958/1 (1984)

13 Ruyssevelt P, Martin C J, Russell $\mathrm{K}$ and Watson D M J Private Communications (1985 and 1986)

14 Page J Ket al. A Meteorological Data Base System for Architectural and Building Engineering Designers Handbook Vol. II 1st edn. Algorithms for Climatological Applications (Sheffield: University Dept. of Building Science) (1984)

15 Judkoff R et al. A Methodology for Validating Building Energy Analysis Simulations Solar Energy Research Institute Draft Report TR-2541508 (1983)

16 Spitler J D et al. A Primer on the Use of Influence Coefficients in Building Simulation Proc. Building Simulation'89, Vancouver, Canada pp 299-304 (1989)

17 Anand D K et al. Validation Methodology for Solar Heating and Cooling Systems Energy 4(4) 549-560 (1979)

18 Sullivan H F and Wright J L Recent Improvements to and Sensitivity of VISION, the glazing system Thermal Analysis Program Proc. 12th Passive Solar Conf., Portland, Oregon (1987)

19 Arashteh D et al. Window 2.0 User and Reference Guide Windows and Daylighting Group, LBL, California (1986)

20 McCabe $M E$ and Hill D Field Measurement of Thermal and Solar Optical Properties of Insulating Glass Windows ASHRAE Trans. 93(1) (1987)

21 Klems J H Measurement of Fenestration Performance under Realistic Conditions Proc. Conf. Windows in Building Design and Maintenance Gothenburg (1984)

22 Mahajan B M National Bureau of Standards Passive Solar Test Facility - Instrumentation and Site Handbook Report NBSIR 84-2911 (1984)

23 Birch J Solar Energy Research Institute Validation Test Cell Site Handbook Solar Energy Research Institute Report 254/9-2-7 (Private communication) (1985)

24 Birch J Solar Energy Research Institute Validation Test House Site Handbook Solar Energy Research Institute Report (Rough draft; Private communication) (1985)

25 Lomas K J et al. Applicability Study 1: A UK Initiative to Determine the Error Characteristics of Detailed Thermal Simulation Models Proc. 2nd European Conf. on Architecture, CEC, Paris pp 559-563 (1989) 